\title{
Isolated hepatic tuberculosis: An uncommon presentation of a common culprit
}

\author{
Minal Shastri, Shripad Kausadikar, Jigar Jariwala, Dhaval Dave, Rushad Patell
}

Department of Medicine, Medical College Baroda and SSG Hospital, Vadodara, Gujarat, India

\section{CASE REPORT}

Please cite this paper as: Shastri M, Kausadikar S, Jariwala J, Dave D, Patell RD. Isolated hepatic tuberculosis: An uncommon presentation of a common culprit. AMJ 2014, 7, 6, 247-250. http://doi.org/10.21767/AMJ.2014.2076

\section{Corresponding Author:}

Rushad Patell

32 ALka Society, Akota, Vadodara 390020, Gujarat, India

Email: rushadpatell@gmail,com

\section{ABSTRACT}

Hepatic tuberculosis (HTB) is commonly encountered in patients with widespread miliary disease. Isolated affection of the liver is extremely rare. We present a case of a young woman who presented with a subacute afebrile hepatic failure. Investigations including a liver biopsy proved that the presentation was due to granulomatous hepatitis secondary to mycobacterial infection of the liver. It is important that tuberculosis (TB) be kept in mind especially in endemic areas even in atypical clinical scenarios by clinicians, radiologists, and pathologists. Use of anti-tuberculous drugs in such cases is usually successful and must be instituted early.

\section{Key Words}

Isolated Hepatic Tuberculosis, Mycobacterium tuberculosis, extra pulmonary tuberculosis

\section{Implications for Practice:}

\section{What is known about this subject?}

Isolated HTB is a rare entity, which requires a high index of suspicion especially in endemic areas. Early diagnosis and treatment with appropriate antimicrobials are effective.

\section{What is the key finding in this case study?}

Subacute liver failure with transaminitis can be due to granulomatous hepatitis secondary to mycobacterial infection in an immunocompetent host.

\section{What are the implications for future practice?}

TB should be suspected in endemic areas as a cause of unexplained liver failure. Early suspicion can ensure early and successful treatment.

\section{Background}

Tuberculosis (TB) is an ancient disease of global importance today; the Indian subcontinent is estimated to bear 20 per cent of the global burden of this deadly disease. ${ }^{1,2}$ Although it is primarily a lung infection, the disease can affect several organ systems and has gained the reputation of being a great masquerader clinically as well as radiologically. ${ }^{3}$ Tuberculous infection of the liver is uncommon in clinical practice but relatively well described in patients with widespread miliary TB. However, isolated affection of the liver is rare and may be missed. The potential hepatotoxicity of the strongholds of anti-tuberculous regimens makes for an additional problematic therapeutic challenge. We present a case and successful management of a young immunocompetent woman presenting with liver failure who on investigation was diagnosed with isolated HTB. Having a high index of suspicion and pursuing this possibility, even in patients without evidence of TB in other systems, is paramount to avoid missing a deadly yet treatable condition.

\section{Case details}

A 20-year-old woman presented with anorexia, yellowish discoloration of the eyes and urine accompanied by bilateral pedal oedema and abdominal distension. These symptoms were progressive over the course of 15 days. She indicated she had not experienced breathlessness, chest pain, diurnal variation in symptoms, or any gastrointestinal bleeding. A physical examination confirmed marked scleral icterus, pitting pedal oedema and signs of free fluid in the abdomen. There was no evidence of organomegaly. There was no history of substance abuse, multiple sexual partners, or history of liver disease in the family.

Laboratory investigations revealed severe anaemia; haemoglobin $7.9 \mathrm{~g} / \mathrm{dl}(12-14 \mathrm{~g} / \mathrm{dl})$ with indices suggested microcytic hypochromic picture, with a corrected 
reticulocyte index of 2.41 per cent; and target cells on peripheral smear. Erythrocyte sedimentation rate was $20 \mathrm{~mm}$ at the end of the first hour. Other investigations suggestive of liver disease included thrombocytopaenia $(48,000$ normal range $150,000-450,000)$, hyperbilirubinaemia $(8.4 \mathrm{mg} / \mathrm{dl}$; normal range $0-1$, with a direct fraction of $6.0 \mathrm{mg} / \mathrm{dl}$ ), elevated Aspartate Transaminase (260U/L; normal range <49U/L), Alkaline Phosphatase (308U/L: normal range 28-111U/L), hypoalbuminaemia $(2.6 \mathrm{gm} / \mathrm{dl}$; normal range $3.6-4.4 \mathrm{gm} / \mathrm{dl})$, and an elevated prothrombin time (test 20.8 seconds, control 12.5 seconds INR 1.8; normal range <1.2). Analysis of the ascitic fluid was suggestive of a SAAG of 2.1 , with $<150$ cells, predominately mesothelium supporting portal hypertension as the aetiology rather then peritonitis. Markers for viral hepatitis and autoimmune hepatitis were negative.

An abdominal ultrasound confirmed moderate to severe ascites and a mildly enlarged spleen. The liver had a diffusely coarsened echo texture with surface nodularity. Portal vein was mildly dilated. A contrast-enhanced CT scan of the abdomen confirmed a normal-sized liver with an irregular surface and heterogeneous enhancement suggestive of granulomatous infiltration. A percutaneous liver biopsy undertaken after correction of the coagulation profile showed incomplete regenerating nodules with benign hepatocytes arranged around sinusoids, showing granular cytoplasm with a few hepatocytes showing feathery degeneration. Large areas of the biopsy showed multiple confluent epithelioid granulomas with Langerhans' giant cells and a few showed caseating necrosis (Figure 1). Zein Nielsen (ZN) stain showed presence of acid-fast bacilli (Figure 2). There was marked portal fibrosis with bridging porto-portal fibrosis, marked piecemeal necrosis with bridging necrosis (Figure 3). There were no obvious features suggestive of autoimmune hepatitis, fungal elements, viral inclusions, parasites, or malignancy. There was no evidence of TB on the chest X-ray.
Figure 1: $\mathrm{H}$ and $\mathrm{E}$ staining of liver biopsy specimen showing granulamatous inflammation and caseous necrosis with epitheloid cells (a), lymphocytes (b) and langhans multinucleated giant cells(c)

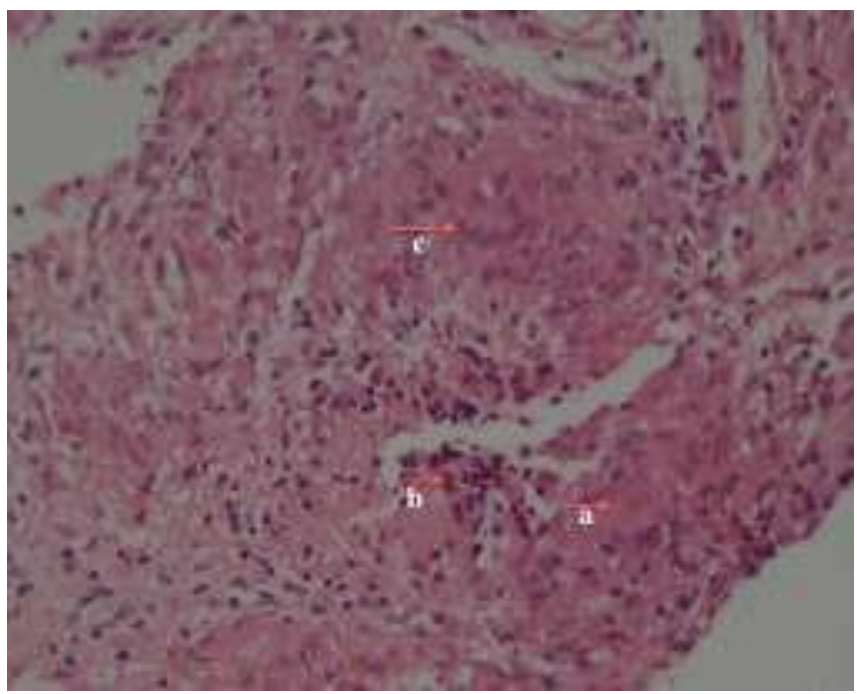

Figure 2: ZN staining of liver biopsy specimen shows acid-fast bacilli

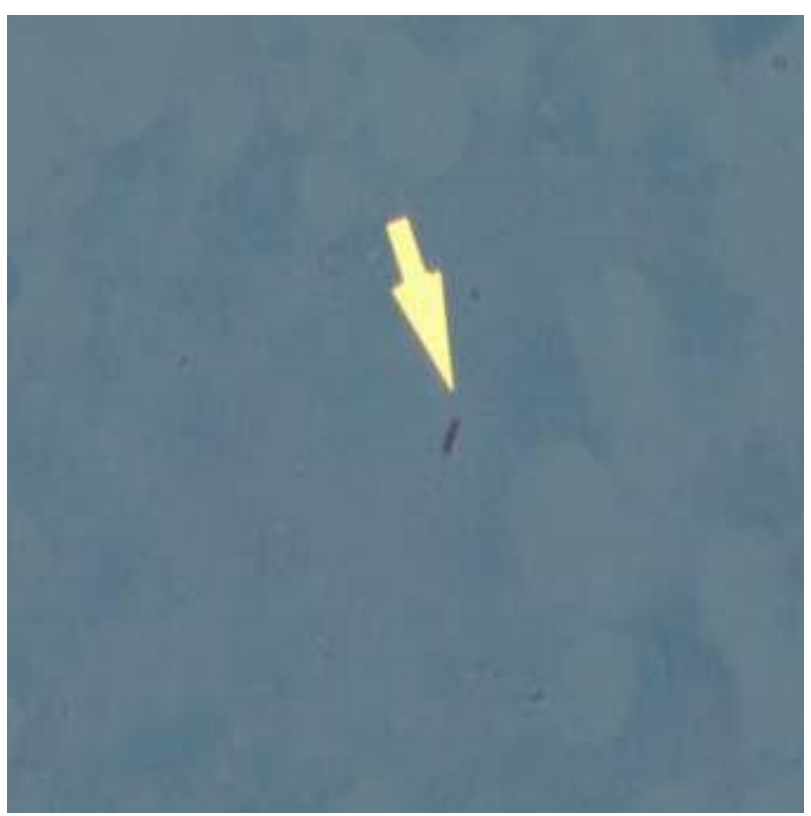


Figure 3 Reticulin staining shows marked fibrosis, with bridging porto portal fibrosis, consistent with stage 3 (precirrhotic/early cirrhosis)

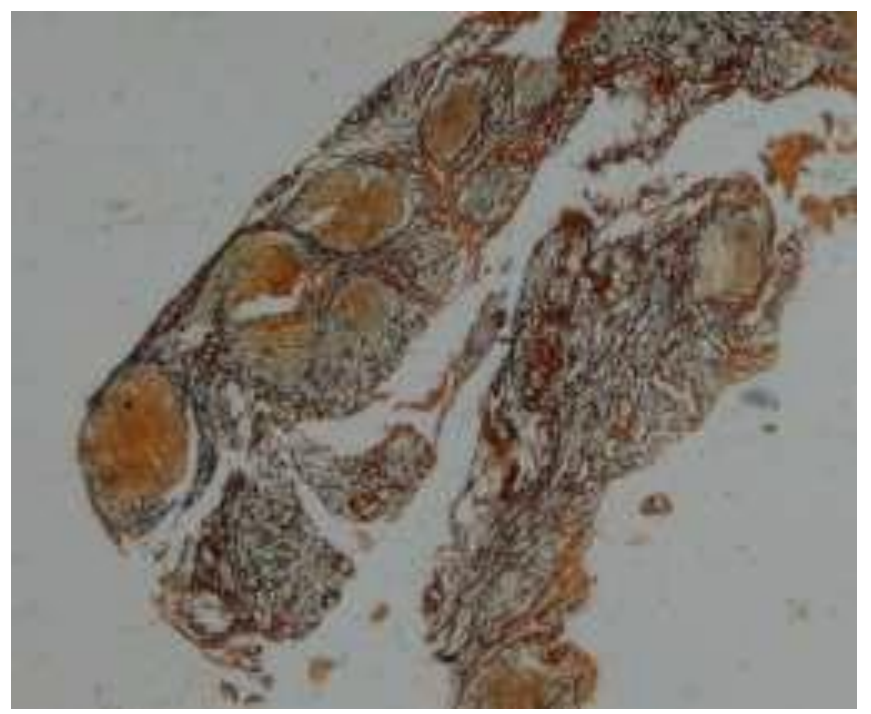

Taking into consideration the severe transaminitis and significant liver compromise (Child Pugh Grade C) it was decided to modify the standard anti-tuberculous therapy (ATT). Initially Ofloxacin, Rifampicin, and Ethambutol were begun and with close monitoring of hepatic profile; Isoniazid and Pyrazinamide were added sequentially. The patient began to improve within two weeks of starting the anti-tuberculous regimen. This was reflected by her improving appetite and decreased ascites and jaundice, as well as the steady decline in serum glutamic pyruvic transaminase (SGPT)/serum glutamic oxaloacetic transaminase (SGOT) and recovery in synthetic functions [albumin and prothrombin timeinternational normalised ratio (PTINR)]. She will continue to receive ATT for one year and be followed up closely.

\section{Discussion}

Although abdominal TB is not uncommon, especially in the tropics, isolated hepatic involvement is extremely rare. A study from a South African general hospital showed that liver TB contributed to 1.2 per cent of the cases of TB diagnosed. ${ }^{4}$ Several forms of HTB are described: ${ }^{5,6,7}$

- most commonly the liver is involved as a part of disseminated or pulmonary TB;

- diffuse involvement (granulomatous hepatitis); and

- granulomas/abscesses, that sometimes need to be differentiated from a neoplasm. ${ }^{8}$

Primary HTB presents several challenges to the clinician. The rarity of the condition and non-specific, scant symptoms make the diagnosis a challenge even in regions where TB is highly prevalent. Demonstration of granulomas on liver biopsy with or without caseation is extremely suspicious, although demonstration of the acid-fast bacilli (AFB) can prove to be difficult. Furthermore, the well-known hepatotoxic potential of several first-line antimicrobials used against TB make therapy a difficult yet imperative challenge.

Correctly diagnosing primary HTB has several pitfalls. Common clinical presentations include fever (70 per cent), weight loss (60 per cent), abdominal pain (50 per cent), hepatomegaly ( 90 per cent), and elevated alkaline phosphatase (80 per cent) ${ }^{4,9}$, In the case described the apparent paucity of constitutional symptoms as opposed to prominent features of advanced liver failure including ascites and pedal oedema is atypical. Unusually raised SGPT/SGOT was also initially misleading. Imaging studies may suggest diffuse nodularity with scattered granulomas and hepatomegaly, ${ }^{9}$ although isolated abscesses or nodules can be confused with primary or secondary malignancy. ${ }^{10}$ Concomitant involvement of the lungs in hepatic TB is very common but as in our case isolated HTB is not common. ${ }^{11,12} \mathrm{~TB}$, particularly extra pulmonary $\mathrm{TB}$, is of growing concern in HIV/AIDS patients; however, our case demonstrates that isolated HTB can occur without any apparent underlying immuno-compromised state.

Granulomatous hepatitis has several important causes, however, relative frequency in the population served is often used as a guide. ${ }^{13}$ Demonstration of AFB on ZN staining and caseous necrosis on biopsy can be very useful pathological tools, but may be absent in a majority of patients and cannot be used to rule out a diagnosis of HTB. ${ }^{4}$ A therapeutic trial of ATT is often warranted empirically especially if steroids are being considered for an alternative diagnosis and the suspicion is high. ${ }^{14}$ It has been recommended that patients with isolated HTB be treated with standard regimens despite the potential hepatotoxicity of the regimen. ${ }^{15}$ Our cases posed an especial challenge due to the relatively atypical transaminitis, a decision to sequentially add Rifampicin, Isoniazid, and Pyrazinamide with close monitoring were made, with a good outcome. HTB is a curable disease with relatively good outcomes; however, a delay in diagnosis can have fatal consequences.

HTB is an important treatable condition especially in populations in the developing world. Increased awareness about its varied presentations, diagnostic pitfalls, and recommended treatment protocols particularly for physicians practicing in tuberculous endemic areas is paramount. The case demonstrates an unusual incident of isolated granulomatous hepatitis in an immunocompetent young woman who presented with liver failure and 
highlights the important learning opportunities achieved in the successful management of the case.

\section{References}

1. World Health Organization. TB/HIV in the South-East Asia Region Status Report. Regional Meeting of National TB Programme Managers WHO/SEARO, New Delhi, India. Geneva: WHO. 2009;Nov 2-5:2-3.

2. Zumla A, Raviglione $M$, Hafner $R$, von Reyn CF. Tuberculosis N Engl J Med. 2013 Feb 21;368(8):745-55. doi: 10.1056/NEJMra1200894.

3. Golden MP, Vikram HR. Extrapulmonary Tuberculosis: an overview. Am Fam Physician. 2005 Nov 1;72(9):1761-8.

4. Essop AR, Posen JA, Hodkinson JH, Segal I. Tuberculosis hepatitis: a clinical review of 96 cases. Q J Med. 1984 Autumn;53(212):465-77.

5. Alvarez SZ. Hepatobilary Tuberculosis. J Gastroenterol Hepatol. 1998 Aug;13(8):833-9.

6. Akçay $\mathrm{MN}$, Polat $\mathrm{KY}$, Oren D, Oztürk G. Primary tuberculous liver abscess. A case report and review of literature Int J Clin Pract. 2004 Jun;58(6):625-7.

7. Farooq Sheikh AS, Qureshi IH, Saba K, Bukhari $\mathrm{MH}$. Primary isolated hepatic tuberculosis. J Coll Physicians Surg Pak. 2013 Feb;23(2):132-6. doi: 02.2013/JCPSP.132136.

8. Zorbas K, Koutoulidis V, Foukas P, Arkadopoulos N. Hepatic tuberculoma mimicking hepatocellular carcinoma in an immunocompetent host. BMJ Case Rep. 2013 Dec 4;2013. pii: bcr2013008775. doi: 10.1136/bcr-2013008775 .

9. Amarapurkar DN, Patel ND, Amarapurkar AD. Hepatobiliary tuberculosis in western India. Indian J Pathol Microbiol. 2008Apr-Jun;51(2):175-81.

10. Huang WT, Wang CC, Chen WJ, Cheng YF, Eng HL. The nodular form of hepatic tuberculosis: a review with five additional new cases J Clin Pathol. 2003 Nov;56(11):8359.

11. Mert A, Ozaras R, Tabak F, Ozturk R, Bilir M. Localized tuberculosis. Eur J Intern Med. 2003Dec;14(8):511-12.

12. Singh $S$, Jain $P$, Aggarwal $G$, Dhiman $P$, Singh $S$, Sen $R$. Primary hepatic tuberculosis: a rare but fatal clinical entity if undiagnosed. Asian Pac J Trop Med. 2012 Jun;5(6):498-9. doi:10.1016/S1995-7645(12)60085-6.

13. Coash M, Forouhar F, Wu CH, Wu GY. Granulomatous liver diseases: a review. J Formos Med Assoc. 2012 Jan;111(1):3-13.

14. Lim EJ, Johnson PD, Crowley P, Gow PJ. Granulomatous hepatitis: tuberculosis or not? Med J Aust. 2008 Feb 4;188(3):166-7.
15. Sonika U, Kar P. Tuberculosis and liver disease: management issues. Trop Gastroenterol. 2012 AprJun;33(2):102-6.

\section{PEER REVIEW}

Not commissioned. Externally peer reviewed.

\section{CONFLICTS OF INTEREST}

The authors declare that they have no competing interests.

\section{PATIENT CONSENT}

The authors, Shastri M, Kausadikar S, Jariwala J, Dave D, Patell R, declare that:

1. They have obtained written, informed consent for the publication of the details relating to the patient(s) in this report.

2. All possible steps have been taken to safeguard the identity of the patient(s).

3. This submission is compliant with the requirements of local research ethics committees. 